\title{
Machine learning robot polishing cell
}

\author{
Max Schneckenburger ${ }^{1, *}$, Luis Garcia ${ }^{1}$, Rainer Börret ${ }^{1}$ \\ ${ }^{1}$ Aalen University of Applied Science, Centre for Optical Technologies, Aalen, 73430, Germany
}

\begin{abstract}
The quality of optical components such as lenses or mirrors can be described by shape errors and surface roughness. With increasing optic sizes, the stability of the polishing process becomes more and more important. Parameters such as chemical stability of the polishing slurry or tool wear are key elements for a deterministic computer controlled polishing (CCP) process. High sophisticated CCP processes such as magnetorheological finishing (MRF) or the zeeko bonnet polishing process rely on the stability of the relevant process parameters for the prediction of the desired material removal. The aim of this work is to monitor many process-relevant parameters by using sensors attached to the polishing head and to the polishing process. Examples are a rpm and a torque sensor mounted close to the polishing pad, a vibration sensor for the oscillation bearings, as well as a tilt sensor and a force sensor for measuring the polishing pressure. By means of a machine learning system, predictions of tool wear and the related surface quality shall be made. The aim is the detection of the critical influence factors during the polishing process and to have a predictive maintenance system for tool path planning and for tool change intervals.
\end{abstract}

\section{Introduction}

With increasing glass optic size, the stability of the polishing process becomes more and more important. The polishing of optical elements depends on several parameters, such as the tilt angle of the tool, the normal force or the friction coefficient, which also influence the material roughness and the material removal rate [1][2][3]. Without controlling the process, the parameters change during the polishing process due to wear. According to the state of the art in the polishing sector, almost no polishing parameters are monitored by sensors. Some parameters such as the normal force or the toolpath velocity are assumed to be constant throughout the polishing process [4]. There will be no stable polishing process on a long period of time without readjusting the polishing parameters. In the past, chemical process conditions assumed to be constant. In the future, additional sensors for measuring chemical values such as temperature, concentration of the polishing slurry and $\mathrm{pH}$ value will be installed. These sensors will obtain a better understanding of the chemical polishing process. As a carrier medium deionized water is used with Ceriumoxid, Opaline from Solvey (former Rhodia) with a diameter of $1 \mu \mathrm{m}$ as polishing grain. The expected $\mathrm{pH}$ value, which is influenced by temperature, will be 7 .

The main part of the setup for this work (cf. Figure 1) is a polishing head designed with the possibility to use either an eccentric pad, a spinning pad or a polishing wheel; therefore, a comparison of these polishing methods is possible. Currently, the setup with the polishing wheel is finished and first tests were performed, while the setups for the pads are still in construction.
Attached to this polishing head are several sensors that monitor the process data and provide information, such as a voltmeter, that measures the power of the motor, a sensor to measure the torque and $\mathrm{rpm}$ of the $\mathrm{pad} / \mathrm{wheel}$, as well as sensors to measure vibrations and the normal force. From the data of the voltmeter and the torque sensor/tachometer, the efficiency of the polishing head can be measured. All sensors are connected to a PLC (Programmable Logic Controller), which collects the data. An $\mathrm{ABB}$ industrial robot is used to perform the movements of the polishing head.

To test the functionality of the polishing head, BK-7 glass samples are used. For the final tests, flat Schott ZERODUR ${ }^{\circledR}\left(\mathrm{Li}_{2} \mathrm{O}-\mathrm{Al}_{2} \mathrm{O}_{3}-\mathrm{SiO}_{2}\right)$ samples with a diameter of $90 \mathrm{~mm}$ and a height of $30 \mathrm{~mm}$ will be polished. Schott ZERODUR ${ }^{\circledR}$ is a common used material for large astronomical mirrors. Furthermore, it is not necessary to monitor the work piece temperature caused by the extremely low coefficient of thermal expansion over a wide temperature range.

For the initial tests with the polishing wheel, the toolpath is programmed to be a straight line forward and backward on the BK-7 glass sample. During the polishing process, the sensor data were monitored and afterwards evaluated using machine learning.

For further testing, the initial surface of the glass will be worked on a lever arm polishing machine to always have the same initial conditions. After a polishing test, the surface can be reset.

\footnotetext{
* Corresponding author: max.schneckenburger@hs-aalen.de
} 


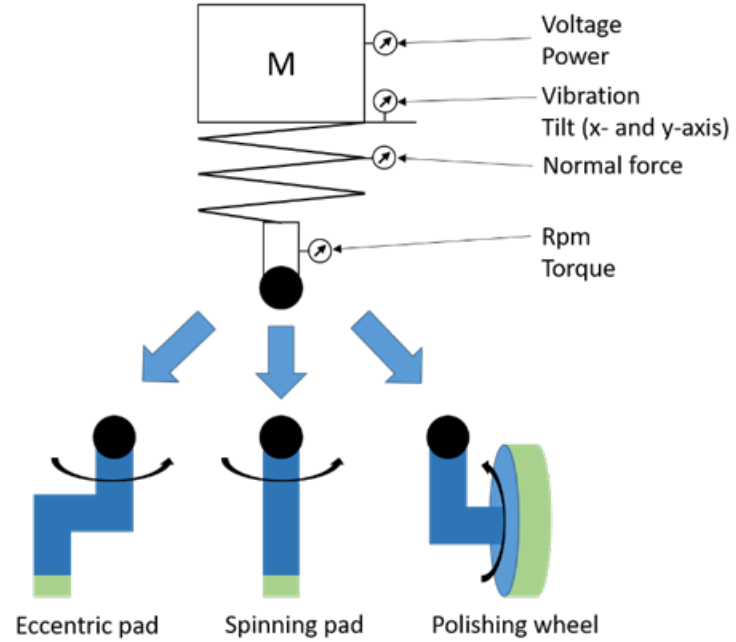

Fig. 1. Principle setup of the polishing head with different sensors and the possibility to use one of three different types of polishing methods (eccentric pad, spinning pad or polishing wheel); the black arrows indicate the movement for each method.

\section{Aim of this research}

The objective of this investigation is to use the knowledge obtained from previous works and improve the surface quality of glass samples, concerning its surface roughness by using machine learning and different polishing modes.

Initial tests have proven the functionality of the sensors, for example, comparative measurements with a highprecision electronic spirit level have verified the repeatability of the tilt sensor.

Data (cf. Figure 2), monitored by the vibration sensor, have shown a wide range of input values instead of the desired constant values. This effect is caused by the instability of the polishing head, but in that case, a stable polishing process was not possible.

In figure 2, the ripple of all sensor data shows the co- and counterrotating during polishing. The small deflections on the ripple of the sensor data (mainly at the normal force and stroke) come from the uneven surface and unbalance of the polishing wheel, caused by the bond seam of the polishing layer. Another future work is a second version of the polishing head with several improvements. One improvement will be the prevention of the oscillations by increasing the stability. Also, more data from state of the art polishing heads are needed to compare typically normal force, oscillation and efficiency values to the new polishing head. A mechanical stop of the z-axis will improve the safety of the polishing head.

In further tests, synchronspeed polishing should be used just with co- or counterrotating of the polishing head.

For the final tests, a DOE by the Taguchi or factorial method will be done to find the correct polishing parameters and to improve the already existing problems. Also, the DOE data will be used to initial train the machine learning algorithm. By doing more experiments and collecting polishing data, machine learning algorithms will be able to do better predictions of the process. Furthermore, the process will be controlled by changing values for normal force, torque and rpm. In combination with CCP and the inline control of the polishing values, there will be an improvement of the surface quality.

The polishing head will be used for shape correction of optics. With the sensors, the polishing head can be controlled and the parameters of the polishing process can be adjusted. This leads to the possibility of achieving a stable polishing process over a longer period of time.

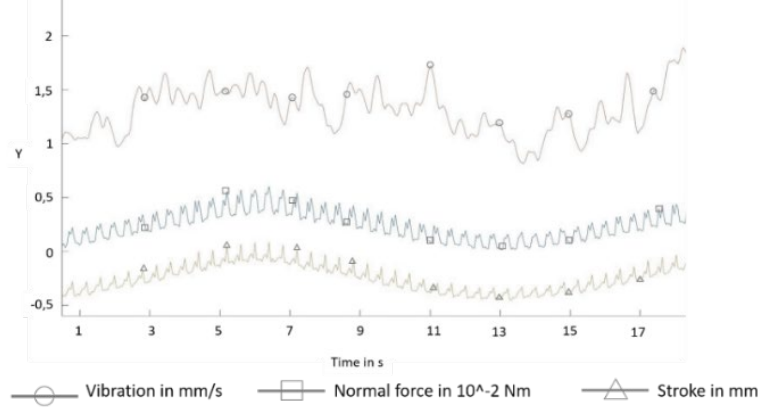

Fig. 2. First sensor data from the polishing head monitored by the PLC (Programmable Logic Controller)

\section{Conclusions}

First results show the functionality of the polishing head, but also some weak points, which have to be improved. On the basis of these initial tests, further experiments have to be performed to study the influence of different process parameters. Monitoring more data over a longer period of polishing time will allow the use of machine learning algorithms, which leads to a better understanding and control of the polishing process.

\section{References}

1. H. Liang, F. Kaufman, R. Sevilla, S. Anjur. „Wear phenomena in chemical mechanical polishing." (1997)

2. J. Luo, D. Dornfeld, „Material Removal Mechanism in Chemical Mechanical Polishing: Theory and Modeling“" (2001)

3. J. Bliedtner, G. Gräfe, ,Optiktechnologie Grundlagen - Verfahren - Anwendungen - Beispiele“, Hanser Verlag (2010), ISBN: 978-3-446-42215-5

4. R. Almeida, R. Börret, D.K. Harrison, A. Desilva, Influence of different polishing materials in the material removal of steel samples (Production \& Manufacturing Research, 2018) 